\title{
Prediction of Flow Pattern Behaviour Behind Square Cylinder using Computational Fluid Dynamic (CFD) Approach
}

\author{
N. $\operatorname{Nordin}^{1 *}$, E. Benard ${ }^{2}$, A. H. A. Kamal ${ }^{1}$, M. T. Ahmad ${ }^{1}$ \\ ${ }^{1}$ Department of Mechanical Engineering, Faculty of Engineering, \\ Universiti Pertahanan Nasional Malaysia (UPNM) Kem, Sungai Besi, 57000 Kuala Lumpur, MALAYSIA
}

${ }^{2}$ ISAE-Supaero, Dept. of Aeronautic and Space Vehicles Design (DCAS), 10 Edouard Belin, 31055 Toulouse Cedex 4, France

DOI: https://doi.org/10.30880/ijie.2020.12.05.017

Received 21 May 2020; Accepted 28 May 2020; Available online 30 June 2020

\begin{abstract}
The aim of this study is to investigate the flow pattern behaviour by using computational fluid dynamic (CFD) approach. The square profile was chosen in purpose to have a better understanding of the behaviour which is relevant to the engineering applications. Numerical simulation was performed on various turbulence models with the range of Reynolds number from 6000 to 80000 with three incidence angles of $0^{\circ}, 15^{\circ}$, and $30^{\circ}$. Mesh dependency study was performed with coarse, base and fine meshes. Fine mesh and standard $\mathrm{k}-\omega$ were chosen as the best meshing and turbulence model to perform the simulation due to the capability in terms of less absolute error on aerodynamic coefficient and clear flow visualisation capture. It was found that the average values of Strouhal number for square profile was 0.12 . For this particular study, the changes of incidence angle and variation of Reynolds number gave a significant flow pattern behind a square profile. The size of the vortices became smaller and closer to the structure as the incidence angle increased. At high Reynolds number, it was also observed that the size of the vortices increased progressively. The prediction of flow pattern behind square cylinder was successfully determined by using CFD approach.
\end{abstract}

Keywords: CFD, URANS, Flow Pattern, Square Profile.

\section{Introduction}

Flow around bluff objects has fascinated considerable attention and has been investigated comprehensively since it is applied in many engineering architectural structures. In aerodynamics, a bluff object is defined as a body, which has a length in the flow direction close or equal to that perpendicular to the flow direction. Bluff body can be characterised as either smooth-edged cross section like circular cylinder or sharp-edged cross section such as rectangular or square cylinder. It is important to understand the flow separation mechanism around the bluff body, so that it will be beneficial into elucidating wind-structure relationship and prediction.

In the case of bluff body with sharp edges, the separation points are fixed to the sharp edges of the body. The behaviour of flow separation on sharp-edged bluff body is depicted in Fig. 1 where the curve lines represent the position of the shear layers [1]. In Fig. 1(a), the smooth and turbulent flows do not re-attach to the surface of the body due to the short width of the body. On the other hand, Fig. 1(b) shows the smooth flow separates from the leading edge does not reattach on the surface of the body but the turbulence flow separates at the leading edge and re-attaches on the surface of the body. Then, the attached flow in the rear part separates again on the sharp trailing edges. This unsteady flow gives rise to the fluctuating drag and lift forces which can stress the body by making it oscillates. This phenomenon also shows that classical aerodynamic concept such as thin airfoil theory is not applicable for this study. It also shows that the geometrical factor, B/D is the main key factor to characterise the flow around sharp-edged bluff body, where B and D denote the width and thickness of the body, respectively [2-3]. 


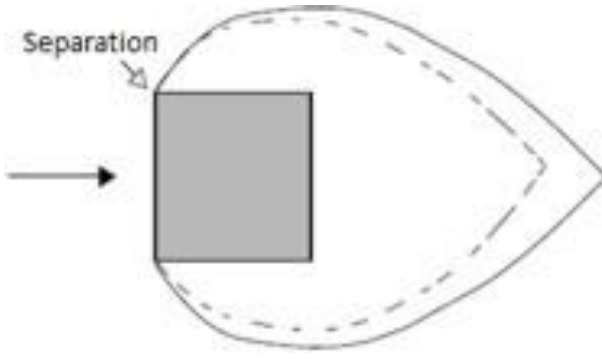

(a) Square cylinder

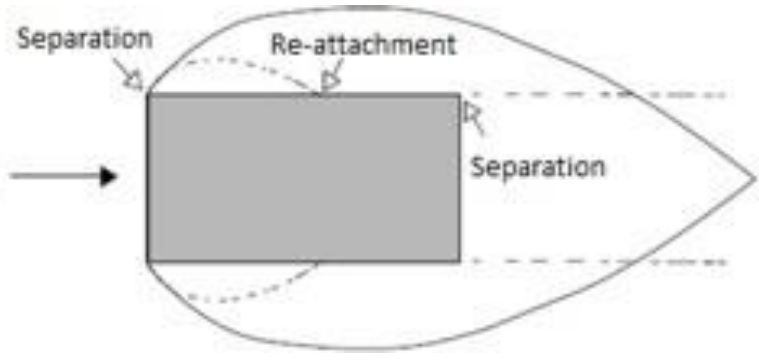

(b) Rectangular cylinder

Fig. 1 - Flow separation on bluff bodies; plain line indicates smooth flow, dash line indicates turbulent flow [1]

The characterisation of flow regimes is expressed by the Reynolds number. Reynolds number is defined as the ratio of inertial forces to viscous forces as shown in Equation (1):

$$
\operatorname{Re}=\frac{V_{\infty} L}{v}
$$

where $V_{\infty}$ is the free stream velocity, $L$ is the reference length and $v$ is the kinematic viscosity of the fluid successfully presented the effect of rectangular aspect ratio in the range of Reynolds number $70 \leq \operatorname{Re} \leq 2 \times 10^{4}$ [2]. It showed the critical value of Reynolds number strongly depends on the aspect ratio of the cylinder when the Reynolds number was less than $5 \times 10^{3}$. Many works have taken place since then, denoting the importance of dimensionless Reynolds number as the main parameter to determine the behaviour of wind-structure system. Depending on the Reynolds number, different flow regimes can be distinguished for a square cylinder starting from low Reynolds numbers. The changes of flow mechanisms on the square profile with respect to the Reynolds number is visualised in Fig. 2 [4]. It shows that, increasing Reynolds number would make the shear layer separates at trailing edge first and two symmetric vortices behind the body can be observed. At the moment, the viscosity magnitude of the flow decreases with the increasing of Reynolds number. When the Reynolds number reaches 60, a separation on the leading edges is observed. This is the range of critical Reynolds number that has been presented by many researchers [2,4-5]. Beyond the critical Reynolds number, the wakes become larger and producing larger scale of shedding vortices.

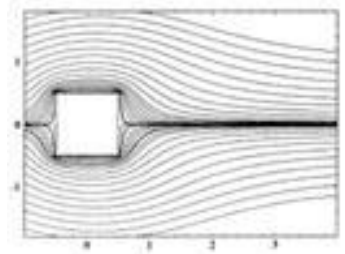

(a) $\mathrm{Re}=1$

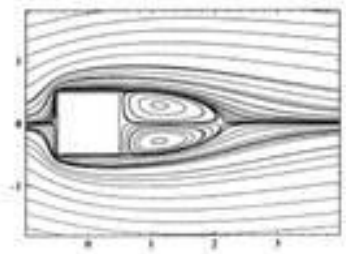

(b) $\mathrm{Re}=30$

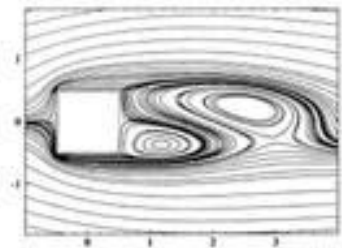

(c) $\mathrm{Re}=60$

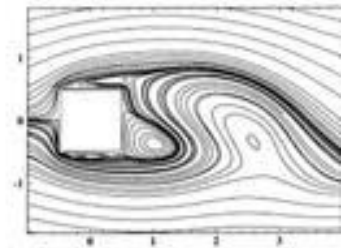

(d) $\operatorname{Re}=200$

Fig. 2 - The effect of streamline around square cylinder with respected to Reynolds number [4]

Another important non-dimensional parameter used to explain the regularity of this wake effect is Strouhal number [6]. Strouhal number used to describe vortex-shedding characteristic as shown in Equation (2):

$$
S t=\frac{f_{s} D}{V_{\infty}}
$$

where $S t$ is the Strouhal number, $f_{s}$ is the frequency of the vortex shedding process, $D$ is the dimension of the body perpendicular to the oncoming flow field, and $V_{\infty}$ is the mean velocity of the uniform flow. This equation indicates that this dimensionless number depends on the body geometry and the Reynolds number. Almost all bluff bodies in cross flows "shed" such vortices at regular rhythms correspond to the respective Strouhal number. It has been recognised as one of the important indices to understand the physical mechanism of aerodynamic instability phenomena. The average value of Strouhal number for square profile is around 0.12-0.13 [7]. Unsteady Reynolds-averaged Navier-Stokes (URANS) based model was used for the simulation work. RANS based model consists of one equation model, two equation model, and Reynolds stress model. There are several criteria that need to be identified to get an accurate result in the simulation: wall $\mathrm{y}^{+}$distribution and time step calculation. 
The first important criteria to determine whether the mesh is fine enough to visualise the boundary layer over an airfoil is the wall $\mathrm{y}^{+}$distribution. The value of wall $\mathrm{y}^{+}$should be approximately less than 1 to capture a good result of the boundary layer [8]. Wall $\mathrm{y}^{+}$is the non-dimensional parameter which is represented in Equation (3):

$$
y^{+}=\frac{h_{1} \underline{U}_{F}}{\vartheta}
$$

where $h_{1}$ is the value of normal cell size of the first cell on the wall, $v$ is the kinematic viscosity, and $U_{F}$ is the friction velocity at the nearest wall. The other important parameter is to determine time step calculation. The simulation requires a small enough time steps to capture the frequency of vortex shedding effectively. FLUENT (2015) recommended to have at least 20-25 time steps in one shedding cycle which is expressed in Equation (4).

$$
\text { Time step size }=\frac{t}{25}=\frac{1 / f}{25}=\frac{\frac{D}{(S t . U)}}{25}
$$

\section{Methodology}

The initial process started with determining the computational domain and boundary condition for the current cases. Meshing procedure was performed using ANSYS ICEM CFD software to select the best meshing for the simulation process. In the simulation setup using FLUENT software, there are several crucial parameters need to be determined: a) time step size, b) mesh convergence study, and c) simulation with different turbulence model. Based on these parameters, the best meshing and turbulence model were selected to continue for different incidence angles [11-12]. Fig. 3 illustrates the flow methodology used for the present study.

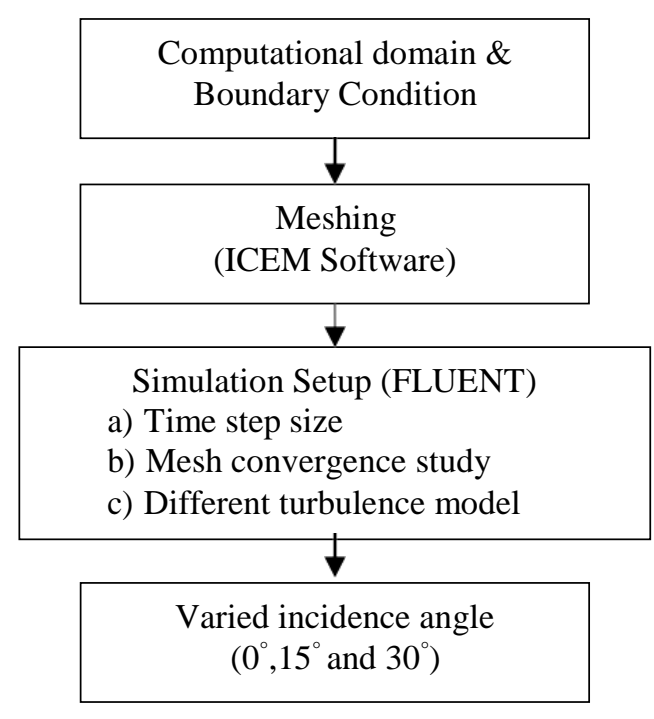

Fig. 3 - Flow methodology of present study

The boundary condition and the computational domain for the simulation of the flow are shown in Fig. 4. The square profile was simulated with a diameter, $\mathrm{D}$ of $40 \mathrm{~mm}$. The computational domain upstream was 8.5 times of the diameter, the downstream was 25 times of the diameter, and 25 times of the diameter for the height of the computational domain.

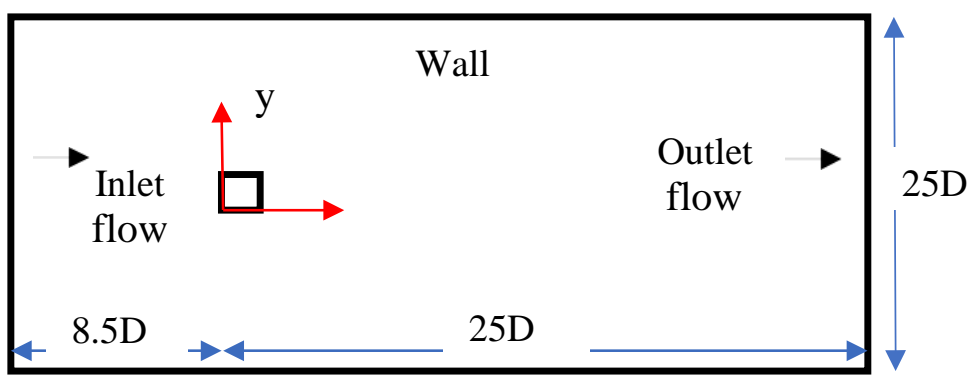

Fig. 4 - Computational domain and boundary conditions 
Boundary conditions were applied to the simulation to obtain the plots of streamlines, vorticity, and velocity magnitude. In the boundary conditions, the free stream velocity was ranged from 2.3 to $29.22 \mathrm{~m} / \mathrm{s}$ which corresponded to the Reynolds number range of 6300-80000, respectively. The outlet boundary was defined with the absolute static pressure 0 Pa. The upper and lower walls were designated to be symmetrical to avoid the effect of shear stress due to wall boundary condition. The square wall was set to default, so it will be considered as no slip condition and effect of wall is minimised. Structured mesh was generated using ANSYS ICEM CFD software. Wall $\mathrm{y}^{+}$value was determined within the range of $0.005-0.045$. This value is valid since it has $y^{+}$value of approximately less than 1 in the viscous sublayer region which viscous stress dominates the wall shear. Fig. 5 shows the mesh generation in the computational domain.

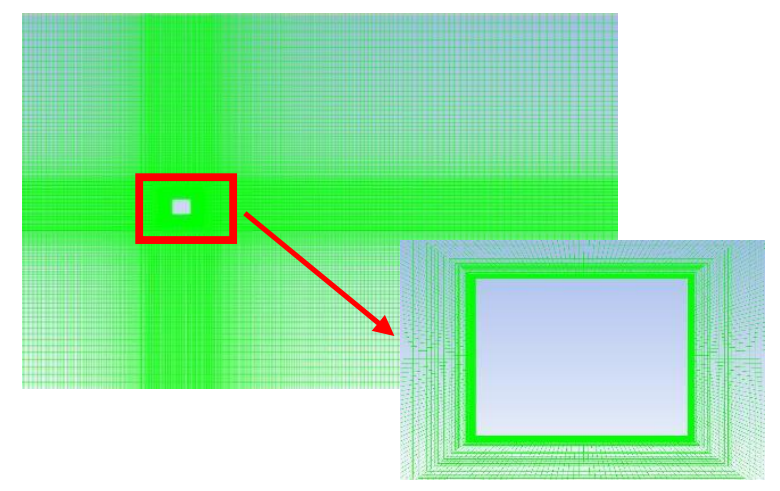

Fig. 5 - Fine mesh around square profile

For the numerical simulation of the flow, the commercial FLUENT software developed by ANSYS was used. This solver helps in solving the RANS equation. To do this, unsteady with pressure based solver was chosen. An unsteady or transient flow was chosen because of the persistent oscillation in the residual data when increasing number of iteration due to the fluctuation of wake velocity in a fairly sinusoidal wave. In unsteady computation, time step size is one of the crucial parameters to be determined to perform a correct computational study. The time steps calculation was already identified in the literature study. Table 1 shows the time steps size at various airspeed with the 25 -time step in one shedding cycle.

Table 1 - Time step size

\begin{tabular}{ccc}
\hline Re. No & Velocity, $\mathbf{~} / \mathbf{s}$ & Time step, $\mathbf{s}$ \\
\hline 6300 & 2.30 & 0.005351 \\
\hline 8000 & 2.92 & 0.004215 \\
\hline 24000 & 8.76 & 0.001405 \\
\hline 50000 & 18.26 & 0.000674 \\
\hline 80000 & $\underline{29.22}$ & $\underline{0.000421}$ \\
\hline
\end{tabular}

In the solution methods, pressure implicit with splitting of operators (PISO) scheme which is useful for unsteady flow with the spatial discretisation was applied using the least square cell based second order upwind scheme to calculate a gradient. A standard solution initialisation was undertaken by computing from all zones. Three types of turbulence models which are standard $\mathrm{k}-\omega$, realizable $\mathrm{k}-\varepsilon$, and re-normalisation group (RNG) $\mathrm{k}-\varepsilon$ were chosen to validate the result obtained from previous paper and select one type of turbulence model for the next analysis with various angle of incidence. For the initial simulation of mesh dependency study, standard $k-\omega$ was selected.

Table 2 - Mesh parameter influences

\begin{tabular}{|c|c|c|c|c|c|}
\hline Mesh & Re.No & Nodes & $\mathbf{C}_{\mathbf{L}(\text { mean })}$ & $\mathbf{C}_{\mathrm{D}(\text { mean })}$ & $\Delta \mathrm{Cd} / \mathrm{Cd}, \%$ \\
\hline \multirow{2}{*}{ Coarse } & 6300 & \multirow{2}{*}{11000} & -0.00572 & 2.15 & 15.6 \\
\hline & 24000 & & -0.00495 & 2.29 & 16.8 \\
\hline \multirow{2}{*}{ Base } & 6300 & \multirow{2}{*}{28000} & -0.01020 & 2.12 & 14.0 \\
\hline & 24000 & & -0.00133 & 2.15 & 9.7 \\
\hline \multirow{2}{*}{ Fine } & 6300 & \multirow{2}{*}{56000} & -0.00032 & 1.64 & 11.8 \\
\hline & 24000 & & -0.00190 & 2.00 & 2.0 \\
\hline \multirow{2}{*}{$\begin{array}{c}\text { Yen \& Yang, } \\
2011\end{array}$} & 6300 & - & -0.02400 & 1.86 & - \\
\hline & 24000 & - & -0.01600 & 1.96 & - \\
\hline
\end{tabular}

To determine the most accurate mesh, three analyses were carried out by modifying the number of cells which are named by coarse, base, and fine using the standard $k-\omega$ turbulence model. The purpose of the grid independence test or 
mesh convergence study is to define the good mesh which were converged to numerical solution data match as close as possible to the experimental data. The detail of the mesh convergence study is as shown in Table 2. Two different Reynolds numbers were chosen similar to the value from the experimental data from Yen \& Yang (2011) to validate present simulations. In this simulation, it was observed that the convergence occurred when the flow field and scalar field were no longer changing. Then, the value can be estimated with the classical criteria of the plateau graph, which are commonly show a decrease in the residual value approximately at $10^{-3}$.

There are no significantly varied of lift coefficient, $C_{L}$ with Reynolds number which the approximation value of $C_{L}$ $=0$ at zero angle of attack because of the symmetrical of the geometry. In term of drag coefficient, $C_{D}$ values, the fine mesh grid generated better results compared to other meshes and much closer to the experimental value with the $2.0 \%$ absolute error at Reynolds number of 24000. From this data, it can be concluded that fine mesh of the square profile can be considered as a good mesh generated to capture aerodynamic coefficient. Another parameter needs to be considered in defining the good mesh visualisation of the boundary layer over the square is the wall $\mathrm{y}^{+}$distribution. In this case study, the values of the $\mathrm{y}^{+}$were in the range of below than 1 which also shows a good agreement with the previous study.

For present study, three different turbulence models were chosen which are standard $\mathrm{k}-\omega$, realizable $\mathrm{k}-\varepsilon$, and $\mathrm{RNG}$ $\mathrm{k}-\varepsilon$. Table 3 shows the computational results between two different turbulence models. In terms of $\mathrm{C}_{\mathrm{D}}$, standard $\mathrm{k}-\omega$ turbulence model was found to be better than realizable $\mathrm{k}-\varepsilon$ and RNG K- $-\varepsilon$ turbulence models with the absolute error of $11.8 \%$ and $2.0 \%$ at Reynolds number of 6300 and 24000, respectively. It is important to note that the $\mathrm{C}_{\mathrm{D}}$ at this level are greatly affected by the boundary layer and the errors were probably from the mesh generated around the boundary layer area and it is not sufficient enough to resolve the boundary layer near the wall of the square profile. In terms of $\mathrm{C}_{\mathrm{L}}$, the value for standard $\mathrm{k}-\omega$ was close to zero at angle $0^{\circ}$.

Table 3 - Parameter analysis with different turbulence model

\begin{tabular}{|c|c|c|c|c|c|}
\hline $\begin{array}{c}\text { Turbulence } \\
\text { Model }\end{array}$ & Re.No & $\begin{array}{l}\text { Velocity, } \\
\text { m/s }\end{array}$ & $\mathbf{C}_{\mathrm{L} \text { (mean) }}$ & $\mathrm{C}_{\mathrm{D}(\text { mean })}$ & $\Delta \mathrm{Cd} / \mathrm{Cd}, \%$ \\
\hline \multirow{2}{*}{ Standard k- $\omega$} & 6300 & 2.3 & -0.00032 & 1.64 & 11.8 \\
\hline & 24000 & 8.76 & -0.0019 & 2.00 & 2.0 \\
\hline \multirow{2}{*}{ Realizable $\mathrm{k}-\varepsilon$} & 6300 & 2.3 & -0.025 & 1.63 & 12.4 \\
\hline & 24000 & 8.76 & -0.02 & 2.016 & 2.9 \\
\hline \multirow{2}{*}{ RNG k-E } & 6300 & 2.3 & -0.005 & 1.572 & 15.5 \\
\hline & 24000 & 8.76 & -0.015 & 2.0296 & 3.6 \\
\hline \multirow{2}{*}{ Shun et. al. } & 6300 & - & -0.024 & 1.86 & - \\
\hline & 24000 & - & -0.016 & 1.96 & - \\
\hline
\end{tabular}

A comparison was also performed in terms of vorticity as shown in Fig. 6 (a), (b), and (c). Flows separation for Fig. 6 (a) and (b) occurred at both upper and lower trailing edges and show continuous vortices shedding. Meanwhile, Fig. 6 (c) shows an attachment at bottom rear surface and there was an interruption in the shedding vortices which gave inaccurate visualisation. In this observation, it shows that standard $\mathrm{k}-\omega$ and realizable $\mathrm{k}-\varepsilon$ were to be more accurate. Considering all the observations, it can be concluded that the standard $\mathrm{k}-\omega$ turbulence model is a better model to address the aerodynamic coefficients. In the case or the vorticity, it can be well addressed using standard $\mathrm{k}-\omega$ and realizable $\mathrm{k}-\varepsilon$ turbulence models.

\section{Result and Discussions}

Several analyses were performed with the varied angle of incidence at the range of Reynolds number starting from 6300 to 80000 . Fine mesh using standard $k-\omega$ turbulence model was used in this study. In the numerical simulation, the Strouhal number was calculated from the result of lift coefficient, $\mathrm{C}_{\mathrm{L}}$ versus flow time. The calculation of the Strouhal number with the timescale of an average of 10 shedding cycle was taken. The vortex shedding frequency is influenced by the distance between two shear layers and airspeed. It was found that the increment distance of two shear layers was caused by the increment on incidence angle. Thus, it will reduce the interaction between the shear layers and reducing the St as well. Based on the vortex shedding frequencies, the respective Strouhal number found in the present simulation was within a range of $0.11-0.14$ which was validated from previous studies.

This simulation generally has shown that the main separation pattern occurs when the Reynolds number or angle of incidence is small. For a square cylinder, the points of separation are fixed at the upstream corner. Fig. 7 shows the development of a vortex shedding where a repetitive pattern of vortices was shed due to the unsteady flow separation. As Reynolds number increases above some critical value, the flow becomes even more turbulent as observed in Fig. 7 (e). At high Reynolds number, it was also observed that the size of the vortices increased progressively. 


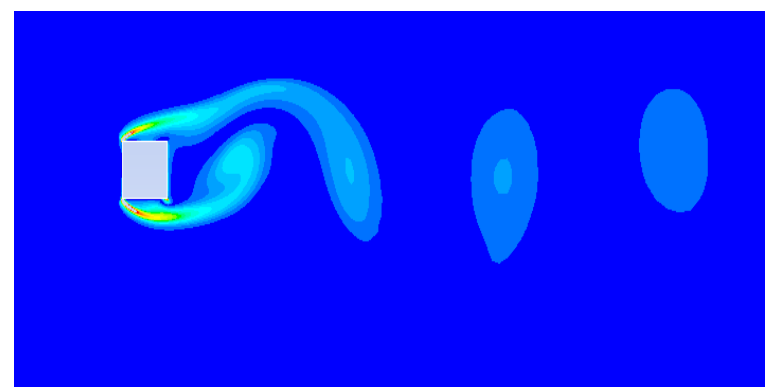

(a) Using Standard k- $\omega$ Turbulence Model

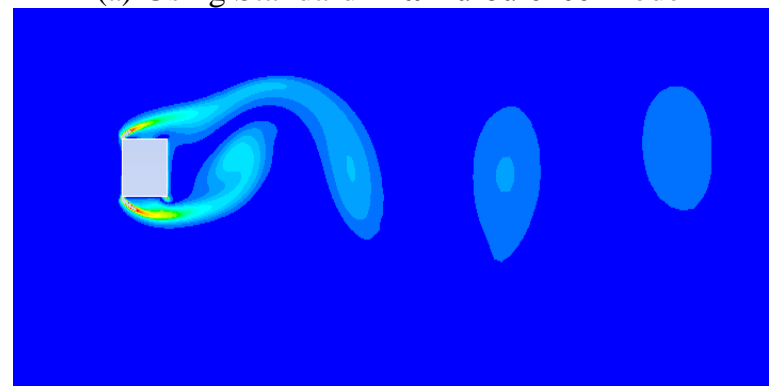

(b) Using Realizable k- $-\varepsilon$ Turbulence Model

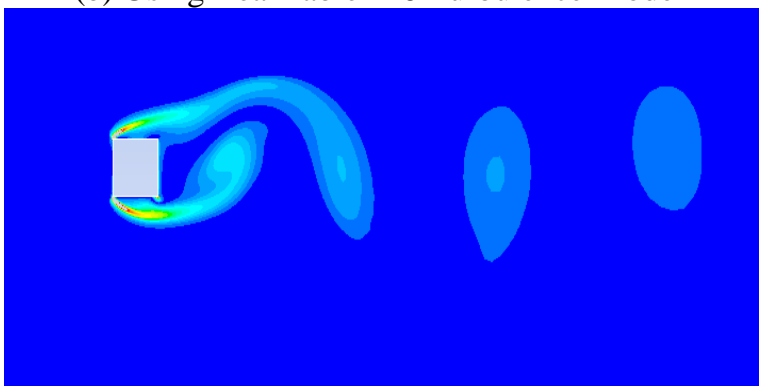

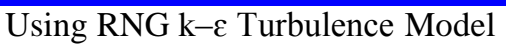

Fig. 6 - Contour of vorticity

The comparison of the vorticity magnitude at the different orientation was observed in Fig. 8 at $\operatorname{Re}=80000$. It was to observe the possibility of shear layer re-attachment over the square surface and the distance over which the shear layer rolls up. At the angle $0^{\circ}$, flow separation was observed from the upstream corner of the square. The shear layer separated in the streamwise direction with the recirculation zone behind. At this angle, the distances over which shear layer rolls up was much longer compared to those at angles $15^{\circ}$ and $30^{\circ}$. Dutta et al. (2008) stated the frequency of the vortex shedding is corresponding to the shear layer roll up vortex over a short distance which causes unsteadiness in the wake. At the angles of $15^{\circ}$ and $30^{\circ}$, flows separation differs between upper and lower surfaces. The shear layer from upper surface diverges away from the square surface while at the lower surface, the shear layer remains close to the square surface. The shear layer on each side for both angles were markedly different with the two vortices shedding frequency. The possibility of unsteadiness in the wake is due to the vortex shedding frequency which corresponds to the rolls up of the vortex at a shorter distance.

Fig. 9 shows the streamline characteristic of the flow at different angles. There were three main flow modes to be highlighted from the analysis for each tested incidence angle. At $0^{\circ}$, the shear layer separation occurred closely to the leading edge of the structure. At $15^{\circ}$, the shear layer separated closely to the leading edge of the structure. Significant separation was observed on the top of the structure but the flow reattached at the bottom of the structure. This reattachment generated a counter-clockwise bubble. At $30^{\circ}$, it was observed that the flow moved along the surface of the structure and did not separate at frontal area of the structure. Fig. 9 (c) exhibits an attached-flow pattern before separation. 


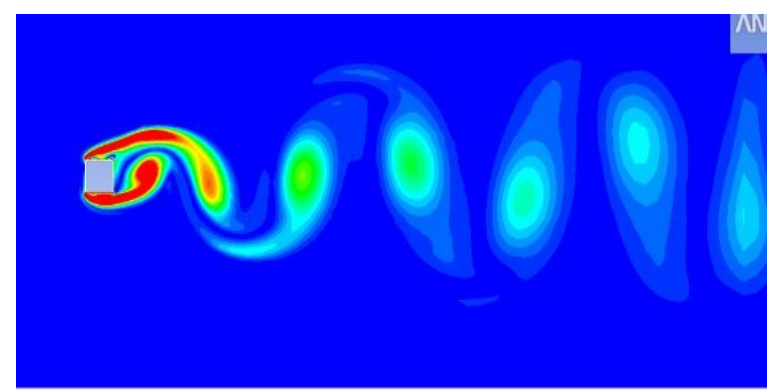

(a) $\operatorname{Re}=6000$

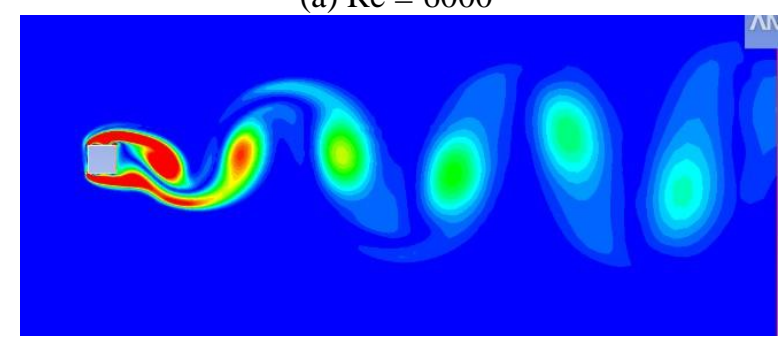

(b) $\operatorname{Re}=8000$

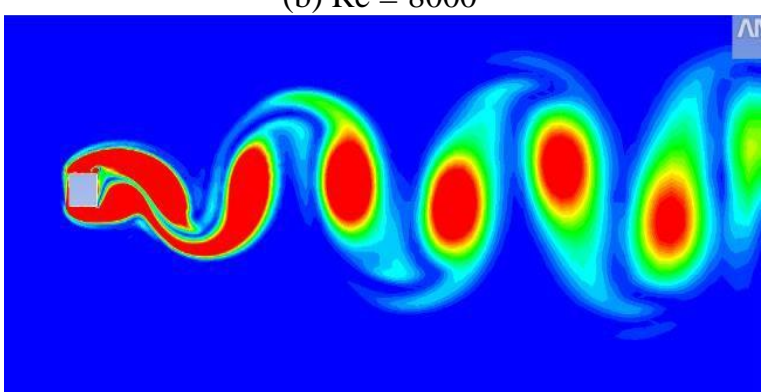

(c) $\operatorname{Re}=24000$

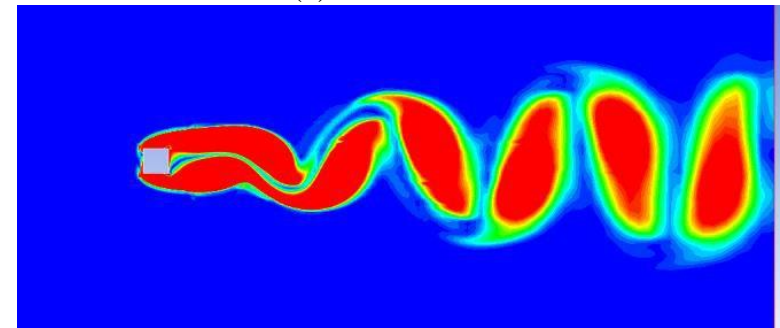

(d) $\operatorname{Re}=50000$

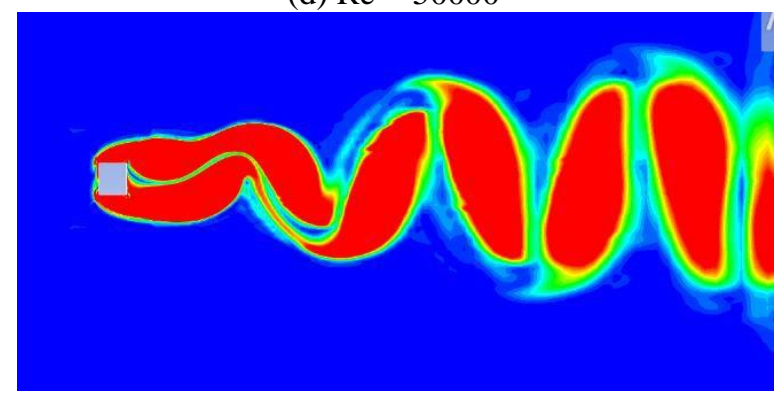

(e) $\operatorname{Re}=80000$

Fig. 7 - Contours of vorticity magnitude at $\mathrm{AOA}=0 \mathrm{0}$ 


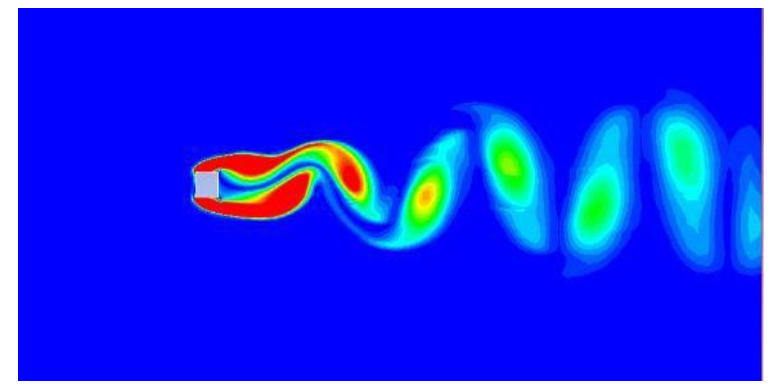

(a) $\mathrm{AOA} 0^{\circ}$

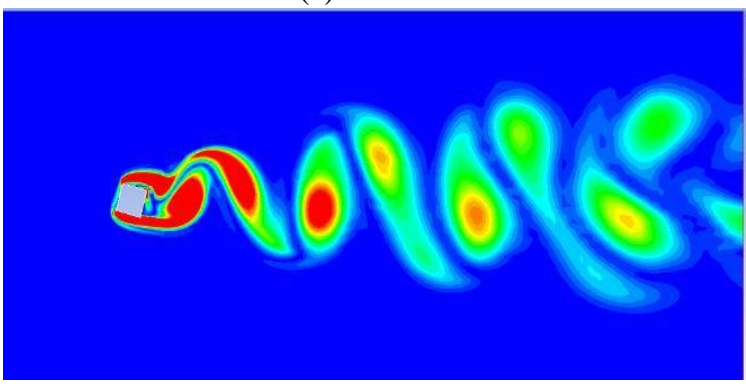

(b) $\mathrm{AOA} 15^{\circ}$

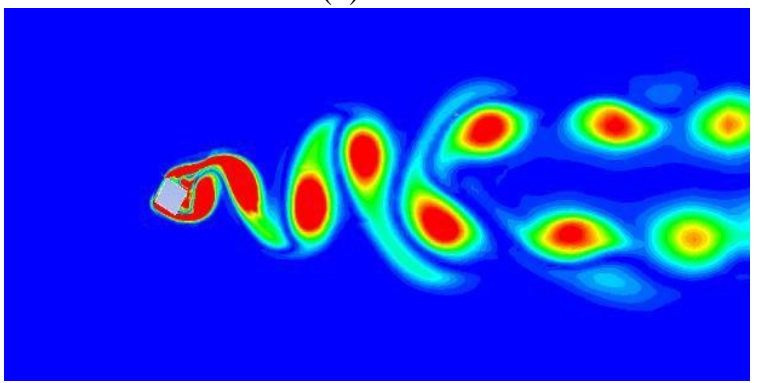

(c) $\mathrm{AOA} 30^{\circ}$

Fig. 8 - Contours of vorticity magnitude at $\mathrm{Re}=\mathbf{8 0 0 0 0}$

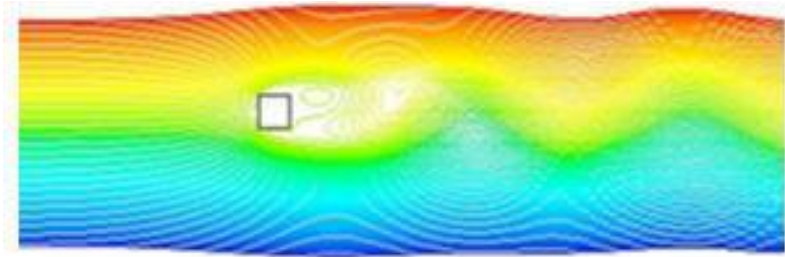

(a) $\mathrm{AOA} 0^{\circ}$

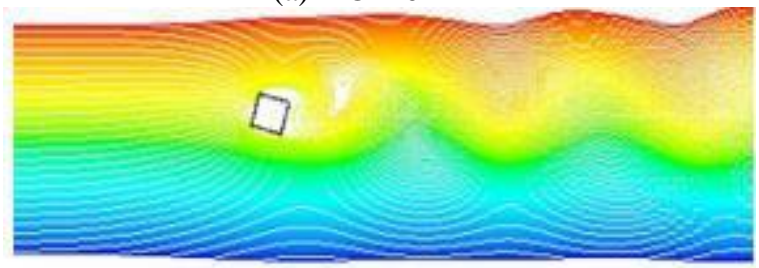

(b) $\mathrm{AOA} 15^{\circ}$

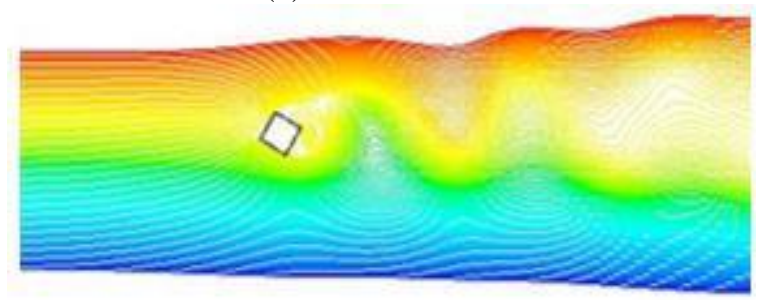

(c) $\mathrm{AOA} 30^{\circ}$

Fig. 9 - Stream function contours at different angle of incidence 


\section{Conclusion}

The simulation work was performed with unsteady RANS in 2D static model. The findings of the simulation are as follow:

a. The standard $\mathrm{k}-\omega$ was chosen to perform the simulation due to the adequacy of the selected criteria during the preliminary analysis; less absolute error on aerodynamic coefficient and provide a clear capture for flow visualization.

b. Strouhal number found at the range of $0.11-0.14$ which are validated with the previous studies.

c. The asymmetry of the shear layer separation due to increment of incidence angle affected the size and the distance of the vortices formation. The size of the vortices became smaller and closer to the structure with the increase of the incidence angle.

\section{Acknowledgement}

The authors would like to express their gratitude and thanks to the Ministry of Higher Education of Malaysia via Universiti Pertahanan Nasional Malaysia (Research funding: FRGS/1/2018/TK03/UPNM/03/1) for funding and supporting this research.

\section{References}

[1] Andrianne, T. (2012). Experimental and Numerical Investigations of the Aeroelastic Stability of Bluff Structures. Department of Aerospace and Mechanics University of Liege.

[2] Okajima, A. (1982). Strouhal Number of Rectangular Cylinders. Journal of Fluid Mechanics, 123, 379-398.

[3] Bearman, P. W., Gartshore, I. S., Maull, D. J., \& Parkinson, G. V. (1987). Experiments on Flow-Induced Vibration of a Square-Section Cylinder. Journal of Fluids and Structures, 1(1), 19-34.

[4] Breuer, M., Bernsdorf, J., Zeiser, T., \& Durst, F. (2000). Accurate Computations of the Laminar Flow Past a Square Cylinder Bases on Two Different Methods: Lattice-Boltzmann and Finitie-Volume. Int. J. Heat and Fluid Flow, 21, 186-196.

[5] Mukhopadhyay, A., Biswas, G., \& Sundararajan, T. (1992). Numerical Investigation of Confined Wakes Behind a Square Cylinder in a Channel. International Journal for Numerical Methods in Fluids, 14(12), 1473-1484.

[6] Dowell, E. H., Scanlan, R. H., Sisto, F., Curtiss, H. C., \& Saunders, H. (1981). A Modern Course in Aeroelasticity. Journal of Mechanical Design.

[7] Dutta, S., Panigrahi, P. K., \& Muralidhar, K. (2008). Experimental Investigation of Flow Past a Square Cylinder at an Angle of Incidence. Journal of Engineering Mechanics, 134(9), 788-803.

[8] Alrutz, T., \& Knopp, T. (2007). Near-Wall Grid Adaptation for Turbulent Flows. International Journal of Computing Science and Mathematics, 1(2/3/4), 177.

[9] FLUENT. (2015). Flow Past a Circular Cylinder.

[10] Yen, S. C., \& Yang, C. W. (2011). Flow Patterns and Vortex Shedding Behavior Behind a Square Cylinder. Journal of Wind Engineering and Industrial Aerodynamics, 99(8), 868-878.

[11] Srivastava, S. (2018). Numerical Simulation of Supersonic Overexpanded Jet from 2-D Convergent-Divergent Nozzle. International Journal of Integrated Engineering, 10(8).

[12] Tham, W. X., Nordin, N., Hariri, A., Nasir, N. F., Mat Isa, N., Yahya, M. N., \& Md. Seri, S. (2019). Asymptotic Computational Fluid Dynamic (ACFD) Study of Three-Dimensional Turning Diffuser Performance by Varying Angle of Turn. International Journal of Integrated Engineering, 11(5), 109-118. 